\title{
Diagnostic Accuracy of Magnetic Resonance Imaging in Anterior Cruciate Ligament Injuries of Knee Joint, in Comparison With Arthroscopy
}

\author{
Authors \\ Dr Shemi Sheriff ${ }^{1}$, Dr Beenamol $S^{2}$, Dr Anas Muhammed ${ }^{3}$ \\ ${ }^{1}$ Resident, Dept of Radiodiagnosis, Govt. Medical College, Thiruvananthapuram \\ ${ }^{2}$ Associate Professor, Dept. of Radiodiagnosis, Govt. Medical College, Thiruvananthapuram \\ ${ }^{3}$ Resident, Dept. of Orthopaedics, Govt. Medical College, Thiruvanathapuram
}

\begin{abstract}
Aim: To determine the accuracy of Magnetic Resonance Imaging in detecting Anterior cruciate ligament injuries of knee, while taking arthroscopy as gold standard.

Methodology: 70 patients referred to the Department of Radiodiagnosis for Magnetic Resonance imaging with clinical suspicion of having ligamentous injuries of knee were included in the study after applying nclusion and exclusion criteria.MR imaging of the knee was done with T2WI,T2WI and PD sequences in axial, sagittal and coronal planes. Arthroscopic correlation and statistical analysis was done for these patients

Results: Magnetic resonance imaging was found to be accurate and non invasive modality for the assessment of ligamentous injuries. The sensitivity, specificity, accuracy, positive predictive value, negative predictive value, positive and negative likelihood ratios of ACL tear were 95\%, 83\%,93\%, 96\%, 77\%, 5.6 and 0.06, Thus, MRI is an appropriate screening tool for therapeutic arthroscopy, and also the test performance is towards diagnostic side than screening, so that it makes diagnostic arthroscopy unnecessary in most of the patients.
\end{abstract}

Keywords: ligament, MRI, arthroscopy,

\section{INTRODUCTION}

ACL injury has an annual incidence of more than 200,000 cases with $\sim 100,000$ of these knees reconstructed annually. ACL reconstructtions are among the most common sports medicine procedures performed. Knee ligament injuries have a tremendous physical and financial impact on the population. Failure to recognize these injuries can lead to complications like an unstable knee, chronic knee pain, and post traumatic arthritis.MRI is the most powerful, accurate and non-invasive method for diagnosing ligament tears. It is more accurate than physical examination and has influenced clinical practice and patient care by eliminating unnecessary diagnostic arthroscopies.

\section{AIMS AND OBJECTIVES}

To determine the accuracy of Magnetic Resonance Imaging in detecting injuries of Anterior Cruciate Ligament of knee, taking arthroscopic findings as gold standard and to know the occurrence of ligament tears following knee injury, o analyse the type and grade of ACL tears with the help of appearances reported in 


\section{JMSCR Vol||05||Issue||01||Page 15373-15378||January}

literature, to validate MRI findings using arthroscopy findings of these patients. And also to study the limitations of MRI in detecting the ACL injury

\section{METHODOLOGY}

Cross-sectional study with - diagnostic test evaluation was done in the Department of Radiodiagnosis, Government medical college, Thiruvananthapuram $\mathrm{f}$ or a period of 18 months from May 2014 to October 2015 on all patients who were clinically suspected of having ligamentous injury of knee and referred to the Department of Radiodiagnosis for Magnetic Resonance imaging.

Inclusion Criteria: Patients who had sustained injuries to either or both knee joints, clinically suspected to have ligamentous injuries and referred for MR imaging of knee joint and was done in our department and subsequently had arthroscopy.

Table 1: Parameters used in MR pulse sequences in our study

\begin{tabular}{|llll|}
\hline Parameters & T1WI & T2WI & PD-FSE \\
TR(ms) & 400 & 4000 & 2200 \\
TE(ms) & 9.5 & 76 & 45 \\
No. of excitations & 2 & 3 & 4 \\
FOV(cmxcm) & $16 \times 16$ & $16 \times 16$ & $22 \times 22$ \\
Matrix(pxp) & $256 \times 192$ & $256 \times 192$ & $320 \times 160$ \\
Slice thickness $(\mathrm{mm})$ & 4 & 4 & 4 \\
Interslice gap $(\mathrm{mm})$ & 0.4 & 0.4 & 0.4 \\
\hline
\end{tabular}

The injuries of anterior cruciate ligament are classified as partial or complete tear for ACL. The presence of coexistent findings like bone injuries, articular cartilage injury, tendon injury, joint effusion, loose bodies or foreign bodies or muscle injuries are also noted. After reaching to a MRI diagnosis, the patients were subjected to arthroscopy of the affected knee. The arthroscopic findings were recorded. Then the findings of MRI and arthroscopy were compared and analyzed. MRI diagnosis was placed into one of the four categories:

\section{Exclusion Criteria}

1) Patients with contraindication to MRI like, metallic implants, cardiac pacemaker sand metallic foreign body in situ.

3) Patients who had prior arthroscopy or surgical intervention to knee joint.4) Patients who are unwilling to participate in the study

Procedure: The subjects who satisfied the inclusion criteria were subjected for MR imaging performed with 1.5T SIEMENS MAGNETOM AVANTO system.MR imaging of the knee was done using a dedicated knee coil with T1 weighted images, $\mathrm{T} 2$ weighted images, and proton density-fat suppressed image in axial, coronal and sagittal planes and medic-sagittal image. The parameters used in MR imaging of the knee are as follows,
2) Patients who are claustrophobic.

1. True positive: When MRI diagnosis of tear was confirmed on arthroscopic evaluation.

2. True negative: If the diagnosis of no tear was confirmed on arthroscopy.

3. False positive: If MRI showed a tear but arthroscopy was negative

4. False negative: If MRI images were negative but arthroscopy showed a tear.

Based on the above categories, sensitivity, specificity, positive predictive value, and negative predictive value were calculated for comparison between MRI and arthroscopy. A p-value less than 0.05 was considered as significant. 


\section{JMSCR Vol||05||Issue||01||Page 15373-15378||January}

DATA MANAGEMENT AND ANALYSIS:

Data Analysis: From the obtained data, the sensitivity, specificity, positive predictive value, negative predictive value, positive and negative likelihood ratios were calculated separately for each ligament by comparison between MRI and arthroscopy. Data analysis was done with the help of Medcalc -15.10.0 and DAG_stat software.

\section{OBSERVATIONS AND RESULTS}

Those patients, for whom arthroscopy was not done or not available were excluded. Thus, out of total 96 patients, 70 were available for analysis finally. Of the total 63 patients were males and 7 females.

Complete ACL tear was seen in $73.7 \%(n=42)$ Partial tear was seen $26.3 \%(n=15)$ Among the MRI reported tears of ACL, complete tears were more common $(74 \%)$, compared to partial tears (26\%). False positive cases (two in number) were partial tears, where hyperintensity was noted due to obliquity of the fibres. Among the false negative cases (three in number), two were noted at the femoral attachment and one tear was noted in mid substance of the ligament.

MRI sequences in detecting ACL tear: Among the 57 cases of ACL tears reported by MRI, the tears were best visualized in $\mathrm{T} 2 \mathrm{~W}$ sagittal sequence in $68 \%$ cases and $\mathrm{T} 2 \mathrm{~W}$ coronal sequence in $25 \%$ cases. Only $7 \%$ tears could be best visualized in PD FSE sagittal sequence.

\section{MRI VS ARTHROSCOPY}

Arthroscopic correlation showed correct identification of ACL tears in 55 out of 57 MRI reported cases with ACL tears. False positive diagnosis were made for 2 cases, which were reported as partial tears. They were not reported as tear by arthroscopy. Out of 13 cases reported not to be having tear by MRI, and in whom subsequent arthroscopy was done due to other reasons like associated meniscal injury, 3 were reported to have tear by arthroscopy. All 3 tears were partial tears, 2 tears noted in femoral attachment and 1 tear in the mid substance of the ligament.

The sensitivity, specificity and accuracy of diagnosing complete ACL tear by MRI were found to be $100 \%$.There were 42 cases of complete ACL tear, reported by MRI. All 42 cases were proven by arthroscopy. There were no false negative cases.

With MR imaging, the sensitivity, specificity and accuracy obtained were $81 \%, 96 \%$ and $93 \%$ respectively. Positive predictive value was about $86.6 \%$. and negative predictive value $94.5 \%$. Likelihood ratio positive and negative were 21.9 and 0.19 , respectively. Accuracy was $93 \%$.

There were 15 cases diagnosed as partial tear by MRI, out of which 13 were confirmed by arthroscopy. There were three false negative cases which were reported as normal by MRI.

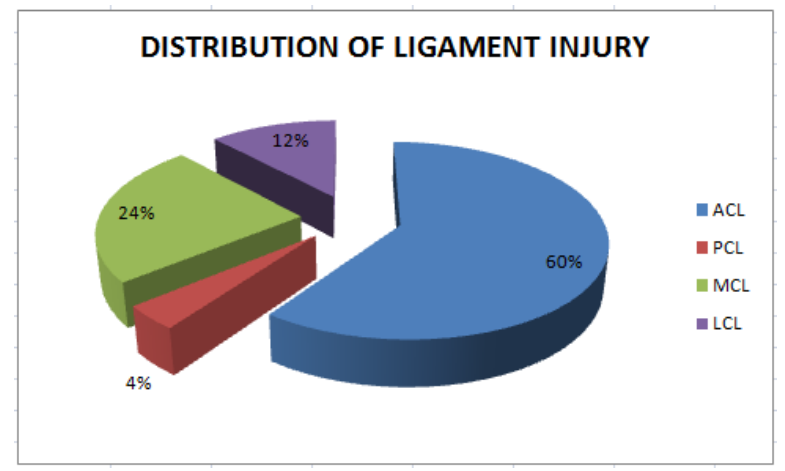

Fig1: Percentage distribution of ligament injuries of knee

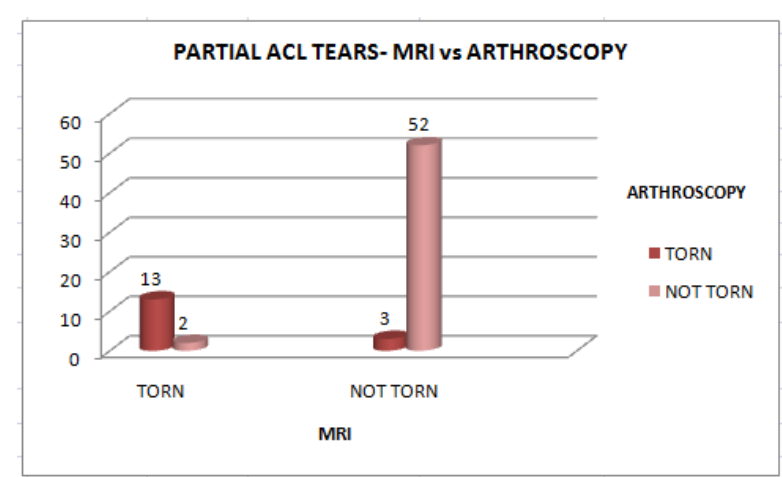

Fig 2 - MR Imaging findings of ACL tears of knee compared to Arthroscopy 


\section{JMSCR Vol||05||Issue ||01||Page 15373-15378||January}

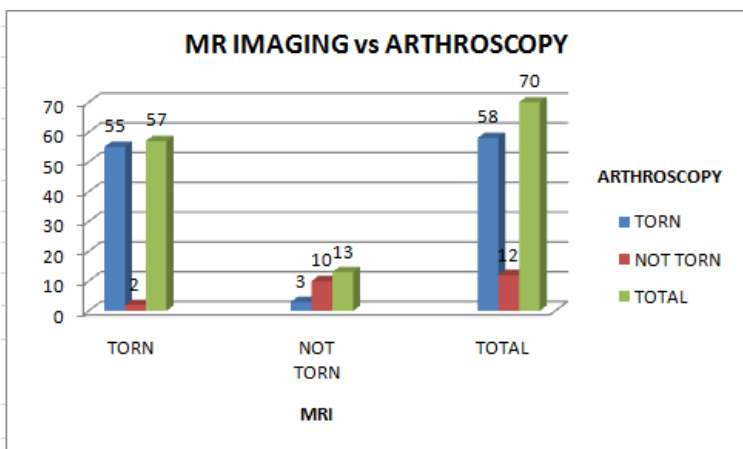

FIG 3 - Complete ACL tear- MRI vs Arthroscopy

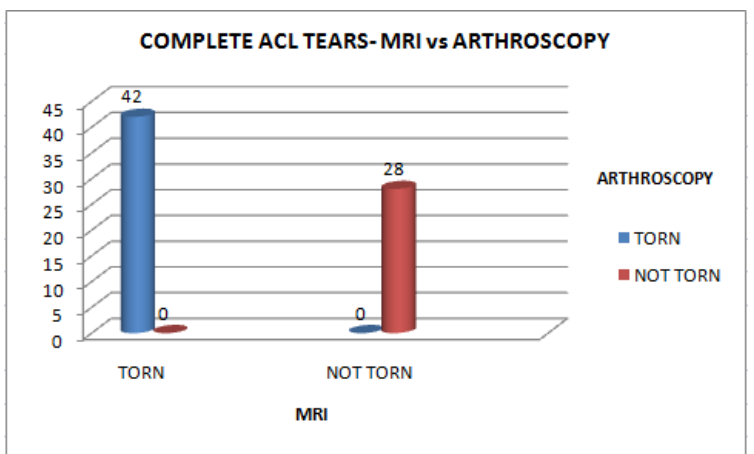

Fig 4- Partial ACL tear - MRI vs arthroscopy

Table 2- Percentage distribution of meniscal tears associated with ACL tear

\begin{tabular}{|c|c|c|c|c|}
\hline Meniscal tear & $\begin{array}{l}\text { Complete ACL } \\
\text { tear }\end{array}$ & Percentage & $\begin{array}{l}\text { Partial ACL } \\
\text { tear }\end{array}$ & Percentage \\
\hline Medial meniscus & 17 & 40.4 & 8 & 53.3 \\
\hline Lateral meniscus & 5 & 12 & 1 & 6.7 \\
\hline No meniscal tear & 20 & 47.6 & 6 & 40 \\
\hline Total & 42 & 100 & 15 & 100 \\
\hline
\end{tabular}
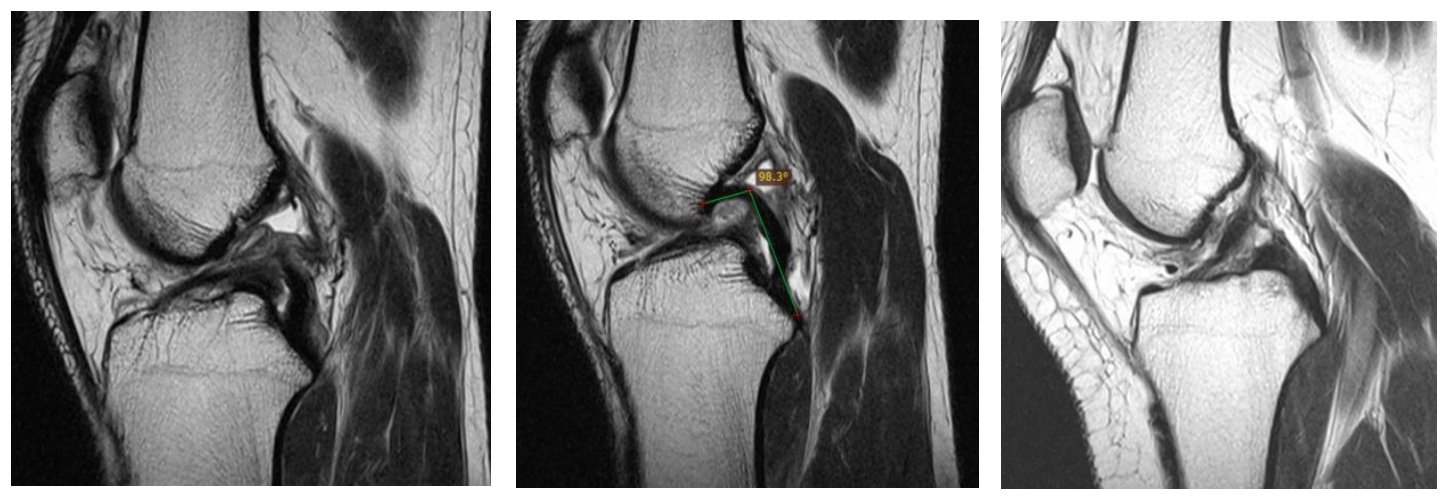

Image 1.- T2W sagittal image, complete ACL tear with non- visualization of fibers. The tear was confirmed by arthroscopy and ACL reconstruction was done. Image 2- T2W sagittal image, showing hyper buckling or "question mark configuration "of PCL which is a secondary sign of ACL tear, Image 3- T2W sagittal image, partial tear of anterior cruciate ligament with ligament laxity. The tear was confirmed by arthroscopy.
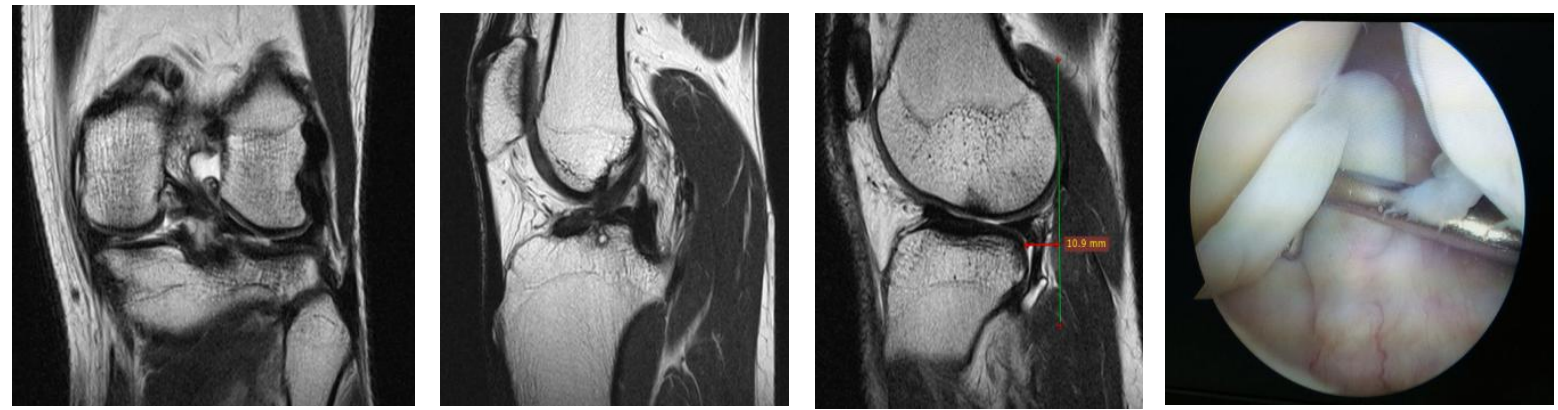

Image 4-T2W coronal image, "empty notch sign" -fluid against interior part of lateral femoral condyle, which is indirect sign of ACL tear. Image 5 - T2W sagittal image, partial ACL tear at the tibial attachment. Fracture noted at intercondylar notch with subchondral cyst. Image 6- T2W sagittal image, A case of complete ACL tear with anterior tibial subluxation, measured at midlateral femoral condyle, which is a secondary sign of ACL tear. Image 7-Torn ACL in arthroscopy 


\section{JMSCR Vol||05||Issue||01||Page 15373-15378||January}

\section{DISCUSSION}

MR imaging has revolutionized knee imaging. It has been compared by various studies between magnetic resonance and arthroscopic findings. These studies validate the role of MR imaging in the clinical arena especially for the evaluation of ligamentous injuries. Out of the 70 subjects, multiple ligament injuries were noted in 23 cases. Hence, the total number of injured ligaments were 96.Of the four major knee ligaments studied, anterior cruciate ligament was more commonly injured (59\% of cases) compared to posterior cruciate ligament $(24 \%)$, medial collateral ligament $(12.5 \%)$ and lateral collateral ligament (4\%).

Of the 57 patients who had anterior cruciate ligament tear, 42(72\%) had complete tear and 16 patients $(27 \%)$ had partial tear. Out of these, only 55 patients identified on MRI were confirmed on arthroscopy. Two patients with positive findings on MRI were found negative on arthroscopy. These were partial tears of ACL cases. Discordant appearance of ACL (when one MR sequence shows disrupted or poorly seen ACL fibres and other sequences show intact ACL fibres) was the reason for this false positive cases ${ }^{1}$.

There are several reasons why the ACL may be abnormal on routine sagittal MR images in absence of ligamentous tear. These include presence of mucoid or eosinophilic degeneration within the ligament, partial volume averaging of the ACL with the lateral femoral condyle or with peri ligamentous fat and suboptimal selection of the sagittal imaging plane to view the ACL incontinuity $^{2}$. In these situations the secondary signs are useful.

The various secondary signs which were seen in ACL injury were buckled PCL, uncovering of the posterior horn of the lateral meniscus and bone contusions in characteristic location. The most common secondary signs which were seen in this study were buckling of PCL and bone contusion.

There were three false negative cases, which were missed on MRI but picked up on arthroscopy. All these cases had partial tear. So, the sensitivity of this test is $95 \%$, specificity is $83 \%$, the positive predictive value is $96 \%$, negative predictive value is $77 \%$ and accuracy is $93 \%$. The positive and negative likelihood ratios were 5.6 and 0.06 , respectively. It is comparable with a previous study of Mink etal ${ }^{3}$ where the reported accuracy of MRI for detecting ACL tear was 95\%. In a study by Singh etal ${ }^{4}$ the sensitivity, specificity and accuracy of MRI in detecting ACL tear was reported to be $98.7 \%, 98.9 \%$ and $98.8 \%$, respectively.

There were 42 cases of complete ACL tear identified on MRI which were confirmed by arthroscopy. Thus the sensitivity, specificity and accuracy of diagnosing complete ACL tears are $100 \%$. Whereas, among 15 cases of partial ACL tears reported by MRI, 13 cases were confirmed by arthroscopy. There were two false positive cases, which were not confirmed by arthroscopy. Three cases of partial tears were diagnosed by arthroscopy, which were missed by MRI. Thus the sensitivity, specificity and accuracy of diagnosing partial ACL tears are $81 \%, 96 \%$ and $93 \%$, respectively. The positive and negative predictive values were 87 and 95, respectively.

The positive and negative likelihood ratios were 21.9 and 0.19 , respectively. It is comparable with a previous study of Umanset $\mathrm{al}^{22}$ which proves that MR imaging has relatively low sensitivity (40\%-75\%) but moderate to high specificity (62\%-94\%) in diagnosis of partial tears.

Among the total 57 cases of ACL tear, 31 cases had associated meniscal tear(54\%). Of which, $71 \%$ had associated medial meniscus tears and $29 \%$ had lateral meniscal tears. Partial ACL tears are observed to have slightly more association with meniscal tears, with about $53 \%$ for medial meniscus and about $6 \%$ for lateral meniscus. Meniscal tears are also noted with complete ACL tears with medial meniscus contributing about $40 \%$ and lateral meniscus $12 \%$.

Different MRI sequences in different planes were used in imaging of the knee. These were T1W, T2W and PD images in coronal, axial and sagittal planes. For ACL tear, only some sequences were considered useful like the sagittal images of T2W, 
coronal $\mathrm{T} 2 \mathrm{~W}$ and proton density sagittal image. Out of these, the most useful sequence was T2WI in sagittal plane ( $68 \%$ of cases). T2W coronal sequence was the second best sequence (in $25 \%$ cases). Only $7 \%$ tears could be best visualized in PD FSE sagittal sequence.

\section{LIMITATIONS OF THE STUDY}

There is a possibility that we have missed a few false negatives, because many of the test negatives did not get gold standard since it is unethical to do arthroscopy in test negatives - a work up bias is likely here. Sample size is small, when statistical analysis is done separately or different ligament tears. A proper blinding of the test result to those performing gold standard test was not practically feasible here. Orthopaedic surgeon had reports of MR imaging available at the time of arthroscopy that may have biased arthroscopic categorization of the tears.

\section{CONFLICT OF INTEREST -None}

\section{REFERENCES}

1. RobertsonL,Schweitzer ME, Bartolozzi $\mathrm{AR}$, et al. Anterior cruciate ligament tears: evaluation of multiple signs with MR imaging.Radiology1994;193:829-834.

2. Ward BD, Lubowitz JH. Basic knee arthroscopy: Part 1-4.Arthrosc Tech. 2013 Nov; 2(4): e497-e499.

3. Heron CW,Calvert PT. Three dimensional gradient echo MRimaging of theknee: comparison with arthroscopy in100 patients. Radiology1992;183:839-842

4. Ruwe Patrick A,Wright James, etal.Tears of the anterior cruciate ligament. Radiology1992;183:335-339.

5. Alex WH, Griffith J, Hung E, Paunipagar B, Law BKY and Yun PSH : Imaging of the anterior cruciate ligament .World $\mathrm{J}$ Orthop. 2011 Aug 18; 2(8) : 75-84 